\title{
On the origin of deleterious mutations
}

A genetic study into Americans of European or African descent finds that the Europeans have a bigger proportion of 'harmful' genes than the Africans. But the conclusion has already been questioned in one of what many expect to be a fresh wave of conflicts over data interpretation as new technologies enable a glut of population-genetics studies.

Carlos Bustamante, a statistical geneticist at Cornell University in Ithaca, New York, and his colleagues compared genetic variation in 20 European Americans and 15 African Americans (K. E. Lohmueller et al. Nature 451, 994-997; 2008), looking specifically at single nucleotide polymorphisms (SNPs) - places where DNA differs between individuals by just one 'letter' of the genetic code. The researchers provide the first unbiased genomewide count of these mutations (previous data sets have not agreed on this number) - it is a significant advance in the field of population genetics.

The findings confirm the idea that after human groups migrated out of Africa, they experienced a population 'bottleneck' in which their overall genetic diversity was reduced. The European population then expanded quickly, accruing new mutations before there was time for the old ones that caused negative consequences to be weeded out. The researchers built computer models to show that their data fit this bottleneck interpretation.

The result is that the European population contains a lower overall genetic diversity than the African population. And a higher proportion of the European genetic diversity is potentially harmful, as the DNA contains mutations that could alter the function of the proteins it encodes, the team says.

However, Alexey Kondrashov, a population geneticist at the University of Michigan in Ann Arbor, says that the data could be interpreted differently - he says that the African and European populations have exactly the same burden of harmful traits.

It all boils down to a small difference in interpretation of the data. The researchers analysed 39,440 SNPs, looking at each one to see whether it matched the ancestral SNP - the one found in the genome of the chimpanzee, the closest genetic relative to humans. The team then examined those found to be non-ancestral called derived SNPs - to determine whether the change had affected the identity of the amino acids (each made of up of three DNA letters) they encode. The 'non-synonymous'

\section{"It is difficult to say} how single nucleotide polymorphisms would affect a person's health."

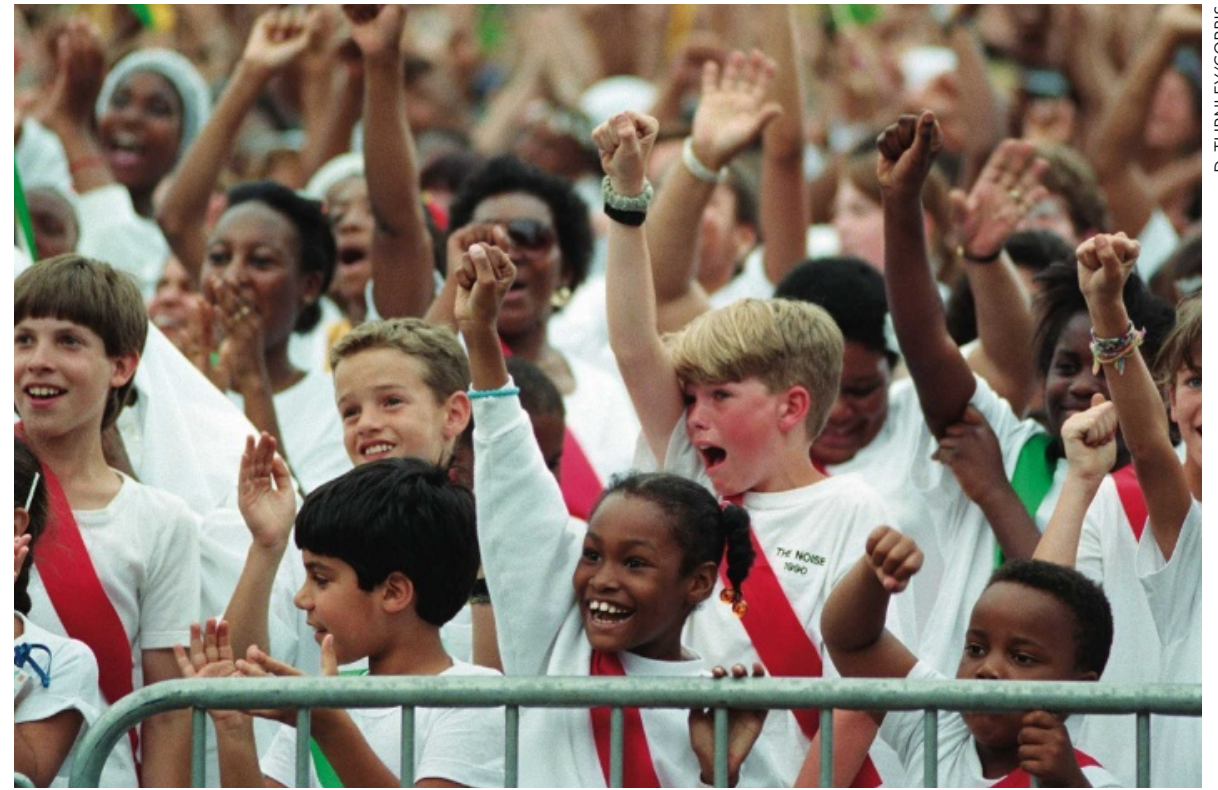

Controversy over data interpretation is likely to increase with the glut of population-genetics studies.

SNPs, the derived SNPs that encoded a different amino acid from the ancestral SNP, were classified by the team as more likely to be damaging. Software was used to classify SNPs as either 'benign', 'possibly damaging' or 'probably damaging' to the proteins they encode.

The team then examined the SNPs unique to each population and found that $47.0 \%$ of the African Americans' SNPs were non-synonymous, compared with $55.4 \%$ of European Americans' SNPs. Of these non-synonymous SNPs, $12.1 \%$ were in the 'probably' damaging bracket for African Americans, compared with 15.9\% in European Americans - a small, but statistically significant difference. "The interesting finding in our paper is that, given lower levels of variation on the whole in Europe than in Africa, a disproportionate amount of [the European variation] is deleterious," says Kirk Lohmueller, a statistical geneticist at Cornell.

However, Kondrashov points out that Bustamante's group also counted the number of non-synonymous SNPs, as well as the 'possibly' and 'probably' damaging SNPs, in the Americans. They found that the European population had about the same number of non-synonymous, possibly-damaging and probably-damaging SNPs as the African American population. These data, as presented in the paper (see Fig. 1, page 995 ) should be interpreted differently, according to Kondrashov. It indicates that the two populations are carrying the same number of potentially deleterious SNPs, he says. "The title of the paper is misleading because they say they showed that Europeans are more genetically burdened than Africans," he says. "But this is simply not true, if you look at Figure 1."

Bustamante disagrees, pointing out that the figure was intended to summarize the genotypes of the individuals in the study - that is, the catalogue of which two SNPs are carried on a person's chromosomes. And, Bustamante says, it is difficult to say how these SNPs would affect a person's health when removed from their genotypic context, so it is not entirely correct to use them as a basis for comparing the overall fitness of the two populations. "We don't interpret Figure 1 to show that individuals in one population or the other are more fit or are more healthy," says Lohmueller.

"We are struggling with the gulf between these statistical descriptions of whole populations and their implications for individual risk," says Andy Clark, a Cornell statistician and one of Bustamante's coauthors. "Just because these patterns exist does not mean any European American individual is going to be at any more risk from disease than any African American." Driving that point home is going to become increasingly difficult - but extremely important - as more population-genetics studies pour out over the coming year.

Erika Check-Hayden 